\title{
The ATLAS muon trigger performance in Run I and initial Run II performance
}

\author{
Rafal Bielski* on behalf of the ATLAS Collaboration \\ University of Manchester, United Kingdom \\ E-mail: rafal.bielski@cern.ch
}

Events with muons in the final state are an important signature for many physics topics at the Large Hadron Collider. An efficient trigger on muons and a detailed understanding of its performance are required. In 2012, the last year of Run I, the instantaneous luminosity reached $7.7 \times 10^{33} \mathrm{~cm}^{-2} \mathrm{~s}^{-1}$ and the average number of interactions that occur in the same bunch crossing was 21 . The ATLAS muon trigger has successfully adapted to this challenging environment by making use of isolation requirements, combined trigger signatures with electron and jet trigger objects, and by using so-called full-scan triggers, which make use of the full event information to search for di-lepton signatures, seeded by single lepton objects. A stable and highly efficient muon trigger was vital in the discovery of the Higgs boson in 2012 and for many searches for new physics. The performance of muon triggers during the Large Hadron Collider Run I data-taking campaigns is presented, together with an overview and preliminary results of the new muon strategy and algorithms, designed to face the demanding environment, which will be adopted during Run II.

XXVII International Symposium on Lepton Photon Interactions at High Energies

17-22 August 2015

Ljubljana, Slovenia

\footnotetext{
* Speaker.
} 


\section{Introduction}

The ATLAS experiment [1] at the Large Hadron Collider (LHC) is a multi-purpose particle detector with a forward-backward symmetric cylindrical geometry and a near to $4 \pi$ coverage in solid an$\mathrm{gle}^{1}$. It consists of an inner tracking detector surrounded by a superconducting solenoid providing a $2 \mathrm{~T}$ axial magnetic field, electromagnetic (EM) and hadron calorimeters, and a muon spectrometer. The inner tracking detector covers the pseudorapidity range $|\eta|<2.5$ and consists of silicon pixel, silicon micro-strip, and transition radiation tracking detectors. Lead/liquid-argon (LAr) sampling calorimeters provide EM energy measurements with high granularity. A hadron calorimeter (iron/scintillator-tile) covers the central pseudorapidity range $(|\eta|<1.7)$. The end-cap and forward regions are instrumented with LAr calorimeters for both EM and hadronic energy measurements up to $|\eta|=4.9$. The Muon Spectrometer (MS) surrounds the calorimeters and is based on three large air-core toroid superconducting magnets with eight coils each. The magnets' bending power ranges from 2.0 to $7.5 \mathrm{Tm}$. The MS includes a system of precision tracking chambers and fast detectors for triggering.

\section{The ATLAS Muon Spectrometer}

Construction of a robust, high-resolution, largeacceptance muon track detector in the ATLAS experiment was particularly motivated by prompt, high transverse momentum $\left(p_{\mathrm{T}}\right)$ muons being distinctive signatures for many physics processes comprising the core of the ATLAS physics program, including the search for the Higgs boson, measurement of its properties, search for new phenomena and precision measurements of Standard Model. The ATLAS MS, presented schematically in Fig. 1, consists of tracking chambers placed in a toroidal magnetic field and exploiting four different technologies, divided into two

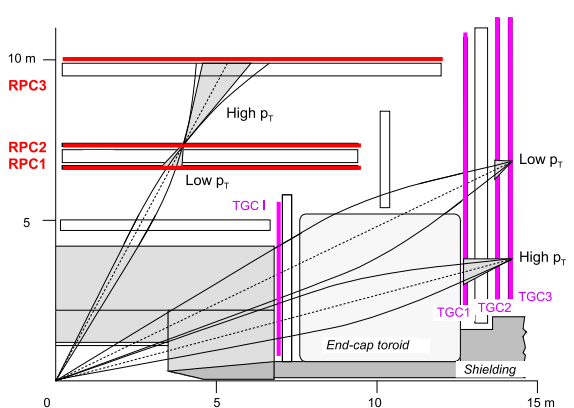

Figure 1: Layout of the ATLAS Muon Spectrometer with the trigger chambers marked in red (RPC) and pink (TGC). separate-function classes. The Resistive Plate Chambers (RPC) in the barrel region $(|\eta|<1.05)$ and the Thin Gap Chambers (TGC) in the end-cap region $(1.05<|\eta|<2.4)$ ) are used to trigger events with high- $p_{\mathrm{T}}$ muons. The Monitored Drift Tubes (MDT) and the Cathode-Strip Chambers (CSC) provide a precision-measurement of muon momenta.

\section{The ATLAS Trigger and Data Acquisition system}

With a $20 \mathrm{MHz}$ (40 MHz by design) pp collision rate ATLAS needs a fast and efficient Trigger and Data Acquisition (TDAQ) system (Fig. 2) able to reduce the throughput to the achievable recording

\footnotetext{
${ }^{1}$ ATLAS uses a right-handed coordinate system with its origin at the nominal interaction point (IP) in the centre of the detector and the $z$-axis along the beam pipe. The $x$-axis points from the IP to the centre of the LHC ring, and the $y$-axis points upwards. Cylindrical coordinates $(r, \phi)$ are used in the transverse plane, $\phi$ being the azimuthal angle around the $z$-axis. The pseudorapidity is defined in terms of the polar angle $\theta$ as $\eta=-\ln \tan (\theta / 2)$. Angular distance is measured in units of $\Delta R \equiv \sqrt{(\Delta \eta)^{2}+(\Delta \phi)^{2}}$.
} 


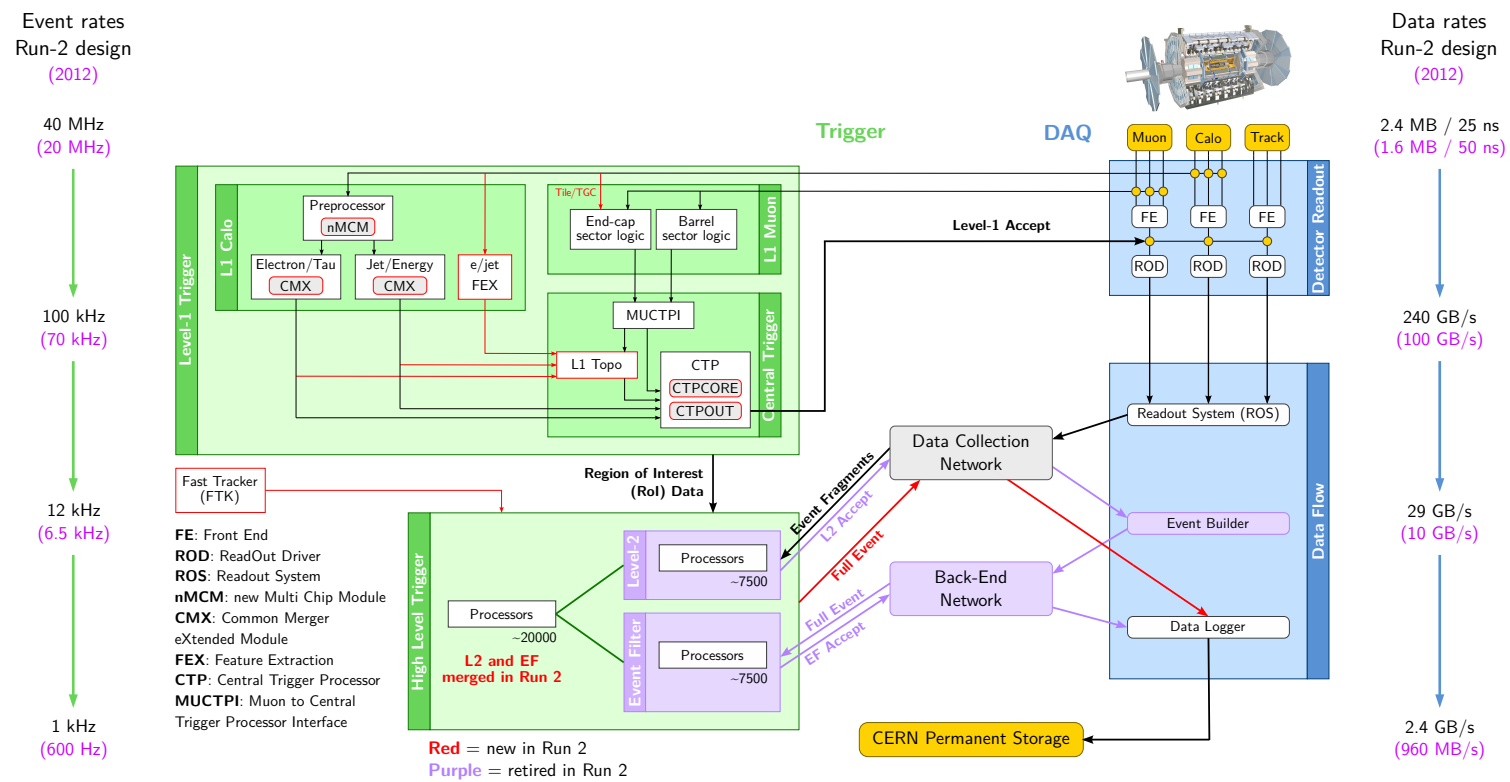

Figure 2: A schematic overview of the TDAQ system in Run I and its modifications for Run II. New elements are marked with red and retired elements with purple.

rate of $\sim 1 \mathrm{kHz}$ by efficiently selecting events with interesting physics signatures. A three-level strategy was designed and employed in Run I. Level-1 (L1) is a hardware-based trigger exploiting custom electronics, with typical Run I output rate of $70 \mathrm{kHz}$. The L1 muon trigger processes only RPC and TGC signals. Level-2 (L2) is a software-based trigger using fast tracking algorithms and processing partial event data from precision chambers around the Region of Interest (RoI) defined by L1. Its typical output rate in Run I was $6.5 \mathrm{kHz}$. Event Filter (EF) is also a software-based trigger, which utilises offline tracking algorithms. It is executed after conversion of full event data to software objects (Event Building). The typical Run I output rate from the EF was $600 \mathrm{~Hz}$.

\section{Muon trigger performance in Run I}

The Run I muon trigger performance was evaluated in data collected in 2012 at $\sqrt{s}=8 \mathrm{TeV}$, corresponding to an integrated luminosity of $20.3 \mathrm{fb}^{-1}$ [2]. The measurement is based on the tag and probe method, typically using the $Z \rightarrow \mu \mu$ decay, with events selected by requiring two reconstructed muons, one of which passes a single muon trigger selection (the tag). The invariant mass of the dimuon system is required to be close to the $\mathrm{Z}$ mass. No other requirements are imposed on the second muon (the probe), thus it is free from trigger bias. The efficiency of a given trigger is calculated as the number of probes passing this trigger divided by the total number of probes. In events where both muons pass the trigger, the second muon is also used as the tag and the first as the probe. Results of this measurement for the logical OR of mu24i and mu36 triggers (selecting single muons with $p_{\mathrm{T}}>24 \mathrm{GeV}$ and an additional track isolation requirement, and selecting single muons with $p_{\mathrm{T}}>36 \mathrm{GeV}$ respectively) are presented in Fig. 3. The efficiency shows a sharp turnon around $p_{\mathrm{T}}=24 \mathrm{GeV}$ and a stable plateau above that value. Measurements in data and Monte Carlo (MC) simulation are consistent with each other. 


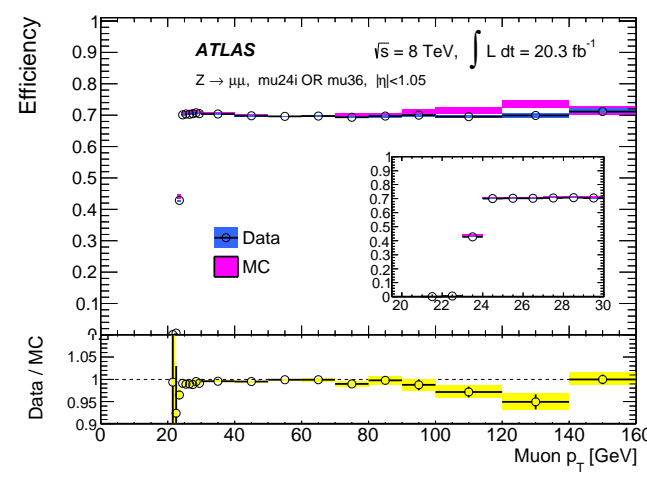

(a)

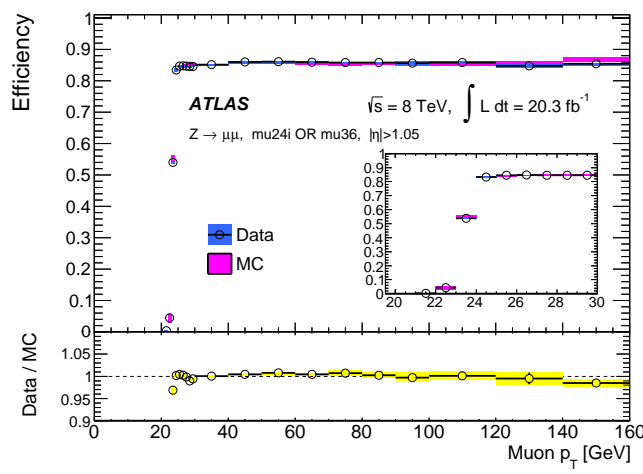

(b)

Figure 3: Efficiency of passing either the mu24i or mu36 chain as a function of the probe muon $p_{\mathrm{T}}$ separately for (a) the barrel region and (b) the end-cap region. The black points represent data, while the purple band represents MC simulation. The lower panel in each figure shows the ratio of the efficiencies of data and MC simulation. The error bars represent the statistical uncertainty, whereas the bands correspond to the combined statistical and systematic uncertainties [2].

\section{Upgrades for Run II}

Event rates expected in Run II will largely exceed typical values observed in Run I. This is driven by the increase of the collision energy from 8 to $13 \mathrm{TeV}$, larger number of proton bunches per fill and tighter beam squeeze (transverse size of the beam at the collision point). Both hardware and software triggers, as well as the data acquisition system underwent a major overhaul in preparation for these new, challenging conditions. Many upgrades were also motivated by inefficiencies or problems emerging over Run I [3].

The L1 trigger now comprises many new purpose-built electronic modules allowing for triggering on new types of signatures, and a new central processor. The main upgrades of the L1 muon system were aimed to resolve problems met in Run I. Twenty new RPC chambers will increase the trigger coverage around the detector support structures, whereas a newly introduced check for coincidence of a signal in the main three TGC layers with a signal in the inner TGC and the Tile calorimeter will allow to reduce the rate of fake muon triggers in the end-caps.

The two software trigger levels were merged into a single High Level Trigger (HLT) and the same algorithms are processed subsequently on a single processing farm with increased computing capabilities. All EF algorithms, including the muon EF, were adapted to process event fragmets requested directly form the readout system.

\section{Initial Run II performance}

The muon trigger efficiency has been studied in the first Run II data collected in July 2015 with $50 \mathrm{~ns}$ bunch spacing and collision energy of $13 \mathrm{TeV}$. The dataset corresponds to an integrated luminosity of $85 \mathrm{pb}^{-1}$. During this data-taking period, several new features of the trigger system were still in a commissioning phase. In particular, the new muon L1 upgrades were not yet operational. 
Nevertheless, the muon trigger system has preserved its high efficiency, rate capability and stability. Figure 4 presents the results of muon trigger efficiency measurement in the end-cap region for the L1 MU15 trigger (L1 trigger selecting single muons with $p_{\mathrm{T}}>15 \mathrm{GeV}$ ) and for a logical OR of two high level triggers - mu20_iloose (seeded by L1 MU15, selecting single $p_{\mathrm{T}}>20 \mathrm{GeV}$ muons with a loose requirement on track isolation) and mu50 (single muons with $p_{\mathrm{T}}>50 \mathrm{GeV}$, seeded by L1 MU20). The HLT efficiency is shown with respect to L1 MU15, showing the excellent performance of the software triggers. The overall efficiency is consistent with results obtained in Run I, proving that the muon trigger maintained its performance after the two-year long shutdown period. The barrel region efficiency is still under study.

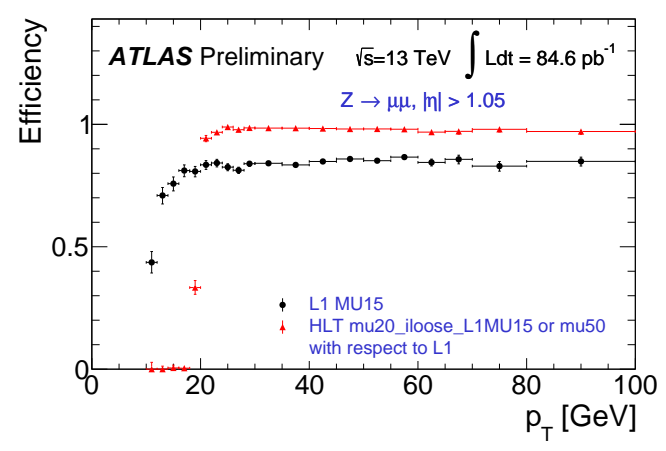

(a)

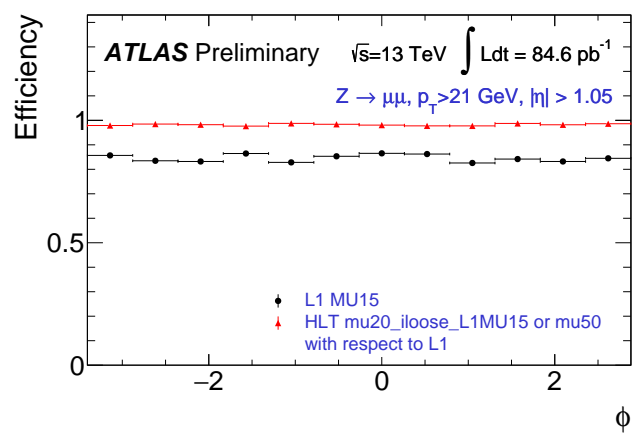

(b)

Figure 4: End-cap muon trigger efficiency as a function of (a) $p_{\mathrm{T}}$ and (b) $\phi$. Black points represent the efficiency of the L1 MU15 trigger with respect to offline reconstructed muon candidates. Red points represent efficiency of the logical OR of mu20_iloose and mu50 triggers with respect to the offline muon candidates that pass the L1 trigger [4]. Only statistical uncertainties are shown.

\section{Summary}

The ATLAS muon trigger system provides a fast and robust selection of muon events at several $p_{\mathrm{T}}$ thresholds and maintains a high and stable plateau efficiency over a wide $p_{\mathrm{T}}$ range, which was evaluated in Run I using the tag-and-probe method. The system underwent and will undergo many upgrades in preparation for high rates expected in Run II. Preliminary studies of the trigger efficiency in the first Run II data collected in July 2015 show that the system preserves its high performance after the two-year upgrade period.

\section{References}

[1] ATLAS Collaboration, The ATLAS Experiment at the CERN Large Hadron Collider, JINST 3 (2008) S08003.

[2] ATLAS Collaboration, Performance of the ATLAS muon trigger in pp collisions at $\sqrt{s}=8 \mathrm{TeV}$, Eur. Phys. J. C75 (2015) 303.

[3] ATLAS Collaboration, Technical Design Report for the Phase-I Upgrade of the ATLAS TDAQ System, CERN-LHCC-2013-018, ATLAS-TDR-023 (2013).

[4] ATLAS Collaboration, Muon trigger performances in 201550 ns data-taking period, https://twiki.cern.ch/twiki/bin/view/AtlasPublic/ MuonTriggerPublicResults\#Plots_for_2015_data_13_TeV. 